\title{
Alterations of Food-specific Serum IgG4 Titers to Common Food Antigens in Patients With Irritable Bowel Syndrome
}

\author{
Hong Sub Lee ${ }^{1,2}$ and Kwang Jae Lee ${ }^{1 *}$ \\ ${ }^{I}$ Department of Gastroenterology, Ajou University School of Medicine, Suwon, Korea; and ${ }^{2}$ Department of Gastroenterology, Myongji Hospital, \\ Goyang; Korea
}

\section{Background/Aims}

The role of dietary factors in the pathogenesis of irritable bowel syndrome (IBS) is still unclear. The aim of this study was to compare IgG4 levels to common food antigens between patients with IBS and healthy controls.

\section{Methods}

Thirty-two patients diagnosed as IBS according to the Rome III criteria (12 diarrhea subgroup; 20 non-diarrhea subgroup) and 32 sex and age-matched healthy controls participated in the study. Serum IgG4 titers to 90 common foods were measured in each subject. The number of subjects with positivity defined as the cut-off value $\geq 0.7 \mathrm{U} / \mathrm{mL}$ was compared.

\section{Results}

Patients with IBS had significantly higher IgG4 titers to wheat, leek and taro compared to those of controls. Serum IgG4 titers to ginger, cocoa, walnut, white radish, onion, and lettuce in IBS patients tended to be higher than controls. IgG4 titers to wheat, gluten and gliadin in the diarrhea subgroup, and lettuce, leek and taro in the non-diarrhea subgroup tended to be higher compared with controls. The number of subjects with positivity to apple, orange, lettuce, and leek was significantly higher in IBS patients than controls. The number of subjects with positivity to apple, orange, gluten, and gliadin in the diarrhea subgroup, and egg white, pineapple, soybean, lettuce, and leek in the non-diarrhea subgroup was significantly higher compared with controls.

\section{Conclusions}

Serum IgG4 antibody levels to some common foods are abnormally elevated in IBS patients. The type of foods with abnormally elevated serum IgG4 titers in the diarrhea subgroup may be different from that in the non-diarrhea subgroup.

(J Neurogastroenterol Motil 2017;23:578-584)

Key Words

Food; Immunoglobulin G; Irritable bowel syndrome

Received: April 19, 2017 Revised: June 26, 2017 Accepted: July 23, 2017

() This is an Open Access article distributed under the terms of the Creative Commons Attribution Non-Commercial License (http://creativecommons. org/licenses/by-nc/4.0) which permits unrestricted non-commercial use, distribution, and reproduction in any medium, provided the original work is properly cited.

*Correspondence: Kwang Jae Lee, MD Department of Gastroenterology, Ajou University Hospital, Ajou University School of Medicine, 164 Worldcup-ro, Yeongtong-gu, Suwon 16499, Korea Tel: +82-31-219-5102, Fax: +82-31-219-5999, E-mail: kjleemd@hotmail.com 


\section{Introduction}

Irritable bowel syndrome (IBS) is a functional gastrointestinal (GI) disorder that has been estimated to affect 1.1-29.2\% of the global population. ${ }^{1,2}$ The pathophysiology of IBS is not completely known. However, several factors, including dietary factors, have been suggested to play a role in the pathophysiology of IBS. About $50 \%$ of patients with IBS report postprandial exacerbations of symptoms. ${ }^{3}$ It has been reported that perceived food intolerance is high $(70 \%)$ in subjects with IBS. ${ }^{4}$ In addition, a prevalence rate of perceived food intolerance is greater than $50 \%$ among subjects with IBS, which is a rate that is 2 -fold greater than that reported by those without IBS. ${ }^{5}$

It is common for IBS patients to avoid some foods, particularly fatty foods, milk products, carbohydrates, caffeine, alcohol, and high protein. ${ }^{6}$ Moreover, they often seek information about dietary modifications. However, because there is little evidence of dietary factors in IBS, physicians usually have difficulty in providing appropriate advice for diet. The estimates of food allergy prevalence were reported to be $8 \%$ of children and $5 \%$ of adults. ${ }^{7}$ Several mechanisms including food hypersensitivity, direct stimulation by food chemicals, and luminal distension have been suggested to explain GI symptoms induced by food. ${ }^{8}$ Among them, food hypersensitivity, classified in IgE-mediated and non-IgE mediated reactions, has been a very controversial subject. ${ }^{9}$ Food-specific $\operatorname{IgE}$ antibodies are known to play a role in food allergy. ${ }^{7}$ However, the role of non- $\operatorname{IgE}$ mediated reaction remains poorly understood.

Among 4 subclasses of IgG antibodies, IgG4 reactions take place in the gut during repeated exposure to food antigens. Studies have reported raised serum IgG4 levels in patients with atopy. ${ }^{10,11}$ Zar et $\mathrm{al}^{12}$ also reported elevated serum IgG4 levels to some foods in IBS patients. In that study, no significant differences in IgE levels were observed between IBS patients and healthy controls. Moreover, it was reported that food elimination based on $\operatorname{IgG} 4$ levels improved symptoms in IBS patients. ${ }^{13}$ However, the role of IgG4 antibodies against foods is controversial yet. ${ }^{14}$ Few studies have evaluated the role of $\mathrm{IgG} 4$ reaction to foods in IBS.

In the present study, we aim to compare food-specific IgG4 levels to common food antigens between patients with IBS and healthy controls.

\section{Materials and Methods}

\section{Study Subjects and Design}

IBS patients who visited the Gastroenterology Department of Myongji Hospital were screened for the current study. All subjects were given the list of symptoms of the validated Korean version of the Rome III questionnaire (Rome III-K). ${ }^{15}$ In addition, blood tests and colonoscopy were performed in all participants.

Exclusion criteria were (1) subjects with organic bowel diseases including inflammatory bowel disease and infectious bowel disease; (2) patients who have functional dyspepsia; (3) patients who have severe comorbidities such as cardiovascular diseases, chronic renal diseases or chronic liver diseases, etc; (4) patients with mental illnesses; and (5) history of GI surgery.

The healthy control group was recruited as the same manner. The control group included healthy subjects who did not have organic diseases and recurrent abdominal symptoms. They were age and sex matched with the patient group. They filled up the symptom questionnaire, and undertook blood tests and colonoscopy in order to check the presence of recurrent abdominal symptoms and organic diseases. All participants gave informed consent before they participated in this study.

This study was performed in a prospective, controlled design. The study protocol and informed consent form was approved by the institutional review board of Myongji Hospital according to the guidelines of the Declaration of Helsinki (MJH14-063). This trial was registered in http://cris.nih.go.kr (KCT0001774). All the participants completed the bowel disease symptom questionnaire (Rome III-K). It was a validated Korean questionnaire of the Rome III criteria.

\section{Measurement of Serum Food Antigen-specific lgG4 Titers}

The eligibility of the subjects was determined before the start of study. Serum food antigen-specific IgG4 titers were measured with the Food Allergy Screening ELISA Kit (Metametrix Clinical Laboratory, 3425 Corporate Way, Duluth, GA, USA). Food allergens are (derived from native foods) immobilized on solid phase (enzyme-linked immunosorbent assay plate), and IgG4 titers to 90 common foods were measured.

Food groups were classified into 6 categories: (1) dairy/meat/ poultry, (2) fruit, (3) fish/shellfish, (4) grains/legumes, (5) vegetables, and (6) nuts/seeds/miscellaneous (Table 1). The antibody 
Table 1. The List of 90 Common Food Antigens Tested in the Current Study

\begin{tabular}{|c|c|c|c|c|c|}
\hline $\begin{array}{l}\text { Dairy, meat, } \\
\text { and poultry antigen }\end{array}$ & Fruit antigen & $\begin{array}{c}\text { Fish and shellfish } \\
\text { antigen }\end{array}$ & $\begin{array}{c}\text { Grains and legumes } \\
\text { antigen }\end{array}$ & $\begin{array}{l}\text { Vegetables } \\
\text { antigen }\end{array}$ & $\begin{array}{c}\text { Nuts, seeds, and } \\
\text { miscellaneous antigen }\end{array}$ \\
\hline Cow's milk & Apple & Cod & Rice & White radish & Almond \\
\hline Casein & Banana & Salmon & Wheat & Cabbage & Coconut \\
\hline Chicken & Grape & Shrimp & Corn & Carrot & Sesame \\
\hline Egg white & Grapefruit & Tuna & Oat & Celery & Walnut \\
\hline Egg yolk & Lemon & Clam & Millet & Cucumber & Cashew nut \\
\hline Lamb & Orange & Crab & Barley & Garlic & Sunflower \\
\hline Pork & Peach & Lobster & Buckwheat & Mushroom & Pepper \\
\hline Beef & Pear & Mackerel & Gluten & Mustard seed & Coffee \\
\hline Yoghurt & Pineapple & Oyster & Gliadin & Olive & Tea \\
\hline Duck & Strawberry & Abalone & Pea & Onion & Baker's yeast \\
\hline \multirow{12}{*}{ Cheese } & Blueberry & Scallop & Soy bean & Green pepper & Ginger \\
\hline & Watermelon & Cuttlefish & Peanut & Potato & Brewer's yeast \\
\hline & Plum & Eel & Kidney bean & Spinach & Cocoa \\
\hline & Kiwi & & & Tomato & Honey \\
\hline & Mango & & & Broccoli & Aspergillus \\
\hline & & & & Lettuce & Kelp \\
\hline & & & & Sweet potato & \\
\hline & & & & Leek & \\
\hline & & & & Taro & \\
\hline & & & & Bamboo shoots & \\
\hline & & & & Eggplant & \\
\hline & & & & Mugwort & \\
\hline
\end{tabular}

titers were expressed as $\mathrm{U} / \mathrm{mL}$. The relative degree of $\mathrm{IgG} 4$ to each food is ranked in classes of 0 to +5 according to cutoff points established by the manufacturer. The cut off value in this study was defined as +2 rank of $0.7 \mathrm{U} / \mathrm{mL}$ for each food antigen. The number of subjects with positivity defined as cut-off value $\geq 0.7 \mathrm{U} / \mathrm{mL}$ was compared using Pearson's Chi-square test.

\section{Sample Size Calculation}

In a previous study, the mean IgG4 level to pork in IBS patients and controls was $31.54 \mathrm{U} / \mathrm{mL}$ (standard deviation 0.45 ) and $30.80 \mathrm{U} / \mathrm{mL}$ (standard deviation 0.59 ), respectively. ${ }^{16,17}$ The number of participants in each arm of this study was calculated to obtain results with an $\alpha$ of 0.05 and a power of 0.90 . Therefore, we calculated that 54 subjects including 27 subjects and 27 controls were needed. We enrolled 70 subjects with the consideration of a $15 \%$ drop out rate.

\section{Statistical Methods}

The statistical comparison between subjects with IBS and healthy control was performed using the Pearson's Chi-square test and an independent sample $t$ test. Odds ratios (ORs) and 95\% con-
Table 2. Demographic Features of the Study Subjects

\begin{tabular}{lccc}
\hline \multicolumn{1}{c}{ Characteristics } & $\begin{array}{c}\text { Patients } \\
(\mathrm{n}=32)\end{array}$ & $\begin{array}{c}\text { Controls } \\
(\mathrm{n}=32)\end{array}$ & P-value \\
\hline Age (age) & $57.4 \pm 11.2$ & $56.8 \pm 14.9$ & 0.865 \\
$\begin{array}{l}\text { Sex (male:female) } \\
\text { History of postprandial }\end{array}$ & $17: 15$ & $17: 15$ & \\
$\quad$ exacerbation $(\%)$ & & 0 & \\
Body mass index $\left(\mathrm{kg} / \mathrm{m}^{2}\right)$ & $24.1 \pm 2.2$ & $23.6 \pm 3.8$ & 0.463 \\
History of diabetes & 5 & 4 & 0.719 \\
History of allergic disease & 1 & 1 & 1.000 \\
\hline
\end{tabular}

Values are presented as mean \pm standard deviation or numbers.

fidence intervals (CIs) were calculated. A $P$-value $<0.05$ was considered significant. SPSS for Windows version 11 software (IBM Corp, Armonk, NY, USA) was used for all analyses.

\section{Results}

\section{Subject Characteristics}

From February 2015 through January 2016, 6 participants of 
the 70 subjects screened were excluded because 1 had ulcerative colitis and 5 subjects withdrew the consent. Among them, $32 \mathrm{pa}-$ tients were diagnosed as IBS using a validated Korean questionnaire of the Rome III criteria (12 diarrhea subgroup; 20 non-diarrhea subgroup). Thirty-two sex and age-matched healthy individuals (15 women and 17 men; mean age, 56 years) without IBS symptoms and organic diseases participated in the study. The demographic features including age, sex, body mass index, and history of allergic disease were comparable in both study groups (Table 2).

\section{Serum IgG4 Titers}

Patients with IBS had significantly higher IgG4 titers to wheat $(4.22 \pm 7.67 \mathrm{U} / \mathrm{mL}$ vs $1.17 \pm 3.06 ; P=0.043)$, leek $(0.13 \pm 0.22$ $\mathrm{U} / \mathrm{mL}$ vs $0.03 \pm 0.10 ; P=0.026)$, $\operatorname{taro}(1.00 \pm 2.17 \mathrm{U} / \mathrm{mL}$ vs $0.11 \pm 0.26 ; P=0.029)$, compared with healthy controls. Serum IgG4 titers to ginger, cocoa, walnut, white radish, onion, and lettuce in IBS patients tended to be higher than those in controls. IgG4 titers to wheat, gluten and gliadin in the diarrhea subgroup tended to be higher than those in controls. Patients in the non-diarrhea subgroup tended to have higher IgG4 levels to lettuce, leek and taro,

Table 3. Food Antigens Showing Differences in IgG4 Titers (U/mL) Between Patients and Controls

\begin{tabular}{|c|c|c|c|c|c|c|c|}
\hline \multirow{2}{*}{ Antigens } & \multirow{2}{*}{$\begin{array}{l}\text { Patients } \\
(\mathrm{n}=32)\end{array}$} & \multirow{2}{*}{$\begin{array}{l}\text { Controls } \\
(\mathrm{n}=32)\end{array}$} & \multirow{2}{*}{$P$-value } & \multicolumn{4}{|c|}{ Subgroups } \\
\hline & & & & Diarrhea & $P$-value & Non-diarrhea & $P$-value \\
\hline Wheat & $4.22 \pm 7.67$ & $1.17 \pm 3.06$ & $0.043^{\mathrm{a}}$ & $5.45 \pm 7.58$ & 0.081 & $3.48 \pm 7.81$ & 0.219 \\
\hline Gluten & $1.23 \pm 2.78$ & $0.47 \pm 1.41$ & 0.173 & $2.00 \pm 2.64$ & 0.079 & $0.77 \pm 2.82$ & 0.608 \\
\hline Gliadin & $1.33 \pm 3.12$ & $0.36 \pm 1.18$ & 0.111 & $2.15 \pm 2.76$ & 0.050 & $0.83 \pm 3.29$ & 0.546 \\
\hline White radish & $0.34 \pm 0.83$ & $0.04 \pm 0.14$ & 0.052 & $0.47 \pm 1.16$ & 0.234 & $0.27 \pm 0.58$ & 0.100 \\
\hline Onion & $0.76 \pm 1.90$ & $0.08 \pm 0.28$ & 0.053 & $0.62 \pm 1.49$ & 0.237 & $0.84 \pm 2.14$ & 0.128 \\
\hline Lettuce & $0.44 \pm 1.10$ & $0.07 \pm 0.16$ & 0.064 & $0.12 \pm 0.22$ & 0.396 & $0.64 \pm 1.35$ & 0.076 \\
\hline Leek & $1.00 \pm 2.17$ & $0.11 \pm 0.26$ & $0.029^{\mathrm{a}}$ & $0.86 \pm 2.38$ & 0.302 & $1.08 \pm 2.10$ & 0.054 \\
\hline Taro & $0.13 \pm 0.22$ & $0.03 \pm 0.10$ & $0.026^{\mathrm{a}}$ & $0.12 \pm 0.23$ & 0.218 & $0.13 \pm 0.22$ & 0.055 \\
\hline Walnut & $0.16 \pm 0.21$ & $0.07 \pm 0.16$ & 0.064 & $0.16 \pm 0.21$ & 0.203 & $0.16 \pm 0.21$ & 0.124 \\
\hline Ginger & $7.19 \pm 2.16$ & $0.59 \pm 1.57$ & 0.095 & $3.62 \pm 1.07$ & 0.349 & $9.33 \pm 2.61$ & 0.151 \\
\hline
\end{tabular}

${ }^{\mathrm{a}}$ Statistically significant.

Values are mean \pm standard deviation unless otherwise stated. Only the variables which have $P<0.1$ was shown in the table.

Table 4. The Number of Subjects with a Positive Antigen Defined as Its IgG4 Titer $\geq 0.7 \mathrm{U} / \mathrm{mL}$ in Irritable Bowel Syndrome Patients and Healthy Controls

\begin{tabular}{|c|c|c|c|c|c|c|c|c|}
\hline \multirow{2}{*}{ Antigens } & \multirow{2}{*}{ Patients } & \multirow{2}{*}{ Controls } & \multirow{2}{*}{ OR $(95 \% \mathrm{CI})$} & \multirow{2}{*}{$P$-value } & \multicolumn{4}{|c|}{ Subgroups } \\
\hline & & & & & Diarrhea & $P$-value & Non-diarrhea & $P$-value \\
\hline Egg white & 11 & 5 & $2.829(0.851-9.402)$ & 0.083 & 3 & 0.473 & 8 & $0.048^{\mathrm{a}}$ \\
\hline Apple & 4 & 0 & & $0.039^{\mathrm{a}}$ & 3 & $0.003^{\mathrm{a}}$ & 1 & 0.202 \\
\hline Orange & 4 & 0 & & $0.039^{\mathrm{a}}$ & 2 & $0.018^{\mathrm{a}}$ & 2 & 0.068 \\
\hline Pineapple & 13 & 7 & $2.444(0.817-7.308)$ & 0.106 & 3 & 0.826 & 10 & $0.035^{\mathrm{a}}$ \\
\hline Gluten & 7 & 4 & $1.960(0.512-7.498)$ & 0.320 & 5 & $0.033^{\mathrm{a}}$ & 2 & 0.784 \\
\hline Gliadin & 6 & 3 & $2.231(0.506-9.835)$ & 0.281 & 5 & $0.013^{\mathrm{a}}$ & 1 & 0.565 \\
\hline Soy bean & 3 & 0 & & 0.076 & 0 & & 3 & $0.024^{\mathrm{a}}$ \\
\hline White radish & 3 & 0 & & 0.076 & 1 & 0.099 & 2 & 0.068 \\
\hline Onion & 5 & 1 & $5.741(0.631-52.234)$ & 0.086 & 2 & 0.112 & 3 & 0.118 \\
\hline Lettuce & 4 & 0 & & $0.039^{\mathrm{a}}$ & 0 & & 4 & $0.008^{\mathrm{a}}$ \\
\hline Leek & 7 & 1 & $8.680(1.001-75.304)$ & $0.023^{\mathrm{a}}$ & 2 & 0.112 & 5 & $0.016^{\mathrm{a}}$ \\
\hline Eggplant & 4 & 1 & $4.429(0.467-42.021)$ & 0.162 & 0 & 0.536 & 4 & $0.045^{\mathrm{a}}$ \\
\hline
\end{tabular}

$\mathrm{OR}$, odd ratio; $\mathrm{CI}$, confidence interval.

${ }^{\text {a }}$ Statistically significant.

Values are presented as numbers unless otherwise stated. Only the variables which have $P<0.1$ was shown in the table. 
compared with healthy controls (Table 3).

\section{The Number of Subjects With Positivity for Serum Food lgG4 Titers}

The number of subjects with positivity for serum food IgG4 titers to apple, orange, lettuce, and leek was significantly higher in IBS patients than that in healthy controls. The number of subjects with positivity to apple, orange, gluten, and gliadin in the diarrhea subgroup, and egg white, pineapple, soybean, lettuce, and leek in the non-diarrhea subgroup was significantly higher, compared with controls (Table 4).

\section{Discussion}

In the present study, we demonstrated that serum IgG4 antibody levels to some foods were abnormally elevated in IBS patients. Furthermore, the type of foods with abnormally elevated IgG4 titers compared to normal controls in the diarrhea subgroup was different from that in the non-diarrhea subgroup. Those findings suggest the possibility that the IgG4-related immune reaction to some foods is involved in the pathogenesis of IBS symptoms.

Previous studies showed that serum IgG4 antibodies to common foods are elevated in IBS patients. ${ }^{16,17}$ Like atopic conditions, those observations suggest the possibility of a role of IgG4-related immune reaction in the pathophysiology of IBS. In a study from the United Kingdom, IBS patients had significantly higher IgG4 titers to wheat, beef, pork, and lamb compared to controls. ${ }^{16}$ In contrast, a study from China showed that IBS patients had significantly higher levels of $\operatorname{IgG}$ antibody to crab, egg, shrimp, soybean, and wheat than controls. ${ }^{17}$ In that study, patients with functional dyspepsia also had significantly higher titers of $\operatorname{IgG}$ antibody to egg and soybean, compared with controls. Those studies suggest that symptoms associated with IBS or functional dyspepsia maybe related to food hypersensitivity. In the present study, IBS patients who overlapped with functional dyspepsia were excluded, because we aimed to investigate the relationship of IgG4-related immune reaction to food antigens with IBS symptoms. We demonstrated that IBS patients had significantly higher IgG4 titers to wheat, leek, and taro compared to controls. Moreover, serum IgG4 titers to ginger, cocoa, walnut, white radish, onion, and lettuce in IBS patients tended to be higher than controls. Although higher levels of IgG4 to wheat antigen were also observed in previous studies, the type of foods showing higher IgG4 titers in IBS patients in the present study was mostly different from that in other previous studies. This difference may be related to the difference in ethnicity or the type of favorite food among countries, and this warrants further investigation.

The level of IgG4 antibodies against foods may just reflect the intake of the food. However, certain foods have frequently been reported to generate or exacerbate IBS symptoms. These foods include wheat, milk products, egg, peanut, soy, sesame, tree nuts, shellfish, spicy foods, coffee, vegetables, fatty foods and alcohol. ${ }^{7,16,17}$ Actually, IBS patients tend to think that specific foods are triggers. ${ }^{18}$ Although many IBS patients mention that they avoid certain foods, this does not seem to significantly affect their intake of nutrients. According to a dietary survey, the real food intake in IBS patients was not significantly different from that in healthy controls. ${ }^{19}$ The observed minor differences show a tendency toward higher intake of fruits and vegetables and a lower intake of meat and dairy products in IBS patients. Therefore, elevation of serum IgG4 titers to some food antigens is more likely to be involved in the pathogenesis or pathophysiology of IBS rather than just reflecting the intake of the food. In the present study, the type of foods with abnormally elevated IgG4 titers compared to normal controls in the diarrhea subgroup was different from that in the non-diarrhea subgroup. This difference may be related to the involvement of IgG4-related immune reaction to specific food antigens in the generation of specific IBS symptoms. This speculation needs to be verified further.

Studies have shown the inflammatory components of IBS. ${ }^{20}$ The difference in the immune reaction to food antigens between individuals may be associated with different permeability to macromolecules in the gut. Actually, increased intestinal mucosal permeability has been reported to be an important pathophysiologic factor in IBS ${ }^{21}$ Increased intestinal mucosal permeability is associated with visceral and thermal hypersensitivity in a subset of IBS patients. ${ }^{21,22}$ Likewise, food hypersensitivity observed in IBS patients may be associated with increased intestinal mucosal permeability. Certain food antigens and specific IgG antibodies may form immune complexes that could cause an inflammatory cascade. ${ }^{23}$ Similarly, high levels of the IgG4 food antibodies are also observed in patients with inflammatory bowel disease, where intestinal mucosal permeability is known to be increased. ${ }^{24,25}$

Some studies have demonstrated that food-specific IgG4 antibody-guided exclusion diet may be effective in alleviating IBS symptoms. ${ }^{26,27}$ The IgG4 antibody-guided exclusion diet affects various symptoms including abdominal pain or discomfort, stool shape, diarrhea frequency, and general feelings of distress. A previous systemic review of clinical trials regarding exclusion diet based on IgG4 showed a $15-71 \%$ response rate. ${ }^{28}$ Immunological adverse reactions by $\operatorname{IgG}$ appear to induce mucosal damage and further increase of mucosal permeability that may be reversed by exclusion 
diet. The positive effect of the IgG4 antibody-guided exclusion diet suggests that the IgG4-related immune reaction to some foods is involved in the genesis of IBS symptoms.

In the present study, IgG4 titers to wheat, gluten, and gliadin tended to be higher in the diarrhea subgroup, whereas those to lettuce, leek, and taro in the non-diarrhea subgroup, compared with controls. The number of subjects with positivity to apple, orange, gluten, and gliadin in the diarrhea subgroup, and egg white, pineapple, soybean, lettuce, and leek in the non-diarrhea subgroup was significantly higher, compared with controls. Those findings indicate that the diarrhea subgroup of IBS may be more associated with wheat and gluten sensitivity than the non-diarrhea subgroup of IBS. Gluten-related disorders include celiac disease, non-celiac gluten sensitivity, and wheat allergy. ${ }^{29,30}$ Non-celiac gluten sensitivity is characterized by intestinal symptoms, such as diarrhea, abdominal discomfort or pain, bloating, and flatulence, or extra-intestinal symptoms such as headache, lethargy, attention-deficit/hyperactivity, skin manifestations, or recurrent oral ulceration. ${ }^{31,32}$ In this condition, gluten-free diet dramatically relieves abdominal pain and diarrhea. Whereas, resumption of a gluten-containing diet induces symptoms again. ${ }^{33,34}$ Gluten influences intestinal barrier functions in IBS patients with diarrhea, and those patients may benefit from a gluten-free diet. ${ }^{34}$ Therefore, the antibody to wheat or gluten seems to play a pathophysiological role in IBS. A different response to food antigens between IBS subgroups needs to be verified in a larger sample of IBS patients. Furthermore, the underlying mechanism related to the association of higher $\operatorname{IgG} 4$ levels to lettuce, leek, taro, egg white, pineapple, and soybean with the non-diarrhea subgroup requires further investigation.

The present study has several limitations. First, the number of subjects who participated in this study may not be sufficient. A larger sample size is required to confirm the results of the present study. Second, there may be a risk of selection bias, because the study population included only the subjects who visited our hospital. Third, the dietary patterns were not checked in the current study. Further studies in the consideration of the dietary patterns are warranted.

In conclusion, serum IgG4 levels to some common foods are abnormally elevated in IBS patients. The type of foods with abnormally elevated IgG4 titers compared to normal controls in the diarrhea subgroup of IBS patients may be different from that in the non-diarrhea subgroup of IBS patients. The role of food hypersensitivity in the pathogenesis of IBS needs further investigation.

\section{Financial support: None.}

\section{Conflicts of interest: None.}

Author contributions: Kwang Jae Lee designed the protocol, conducted the study, collected and interpreted data, and drafted the manuscript; and Hong Sub Lee conducted the study, collected and interpreted data, and drafted the manuscript.

\section{References}

1. Canavan C, West J, Card T. The epidemiology of irritable bowel syndrome. Clin Epidemiol 2014;6:71-80.

2. Oshima T, Miwa H. Epidemiology of functional gastrointestinal disorders in Japan and in the world. J Neurogastroenterol Motil 2015;21:320329.

3. Ragnarsson G, Bodemar G. Pain is temporally related to eating but not to defaecation in the irritable bowel syndrome (IBS). Patients' description of diarrhea, constipation and symptom variation during a prospective 6-week study. Eur J Gastroenterol Hepatol 1998;10:415-421.

4. Monsbakken KW, Vandvik PO, Farup PG. Perceived food intolerance in subjects with irritable bowel syndrome - etiology, prevalence and consequences. Eur J Clin Nutr 2006;60:667-672.

5. Locke GR 3rd, Zinsmeister AR, Talley NJ, Fett SL, Melton LJ. Risk factors for irritable bowel syndrome: role of analgesics and food sensitivities. Am J Gastroenterol 2000;95:157-165.

6. Halpert A, Dalton CB, Palsson O, et al. What patients know about irritable bowel syndrome (IBS) and what they would like to know. National survey on patient educational needs in IBS and development and validation of the patient educational needs questionnaire (PEQ). Am J Gastroenterol 2007;102:1972-1982.

7. Sicherer SH, Sampson HA. Food allergy: epidemiology, pathogenesis, diagnosis, and treatment. J Allergy Clin Immunol 2014;133:291-307.

8. Gibson PR. Food intolerance in functional bowel disorders. J Gastroenterol Hepatol 2011;26(suppl 3):128-131

9. Cuomo R, Andreozzi P, Zito FP, Passananti V, De Carlo G, Sarnelli G. Irritable bowel syndrome and food interaction. World J Gastroenterol 2014;20:8837-8845

10. Gondo A, Saeki N, Tokuda Y. IgG4 antibodies in patients with atopic dermatitis. Br J Dermatol 1987;117:301-310.

11. Noh G, Ahn HS, Cho NY, Lee S, Oh JW. The clinical significance of food specific IgE/IgG4 in food specific atopic dermatitis. Pediatr Allergy Immunol 2007;18:63-70.

12. Zar S, Benson MJ, Kumar D. Food-specific serum IgG4 and IgE titers to common food antigens in irritable bowel syndrome. Am J Gastroenterol 2005;100:1550-1557.

13. Zar S, Mincher L, Benson MJ, Kumar D. Food-specific IgG4 antibodyguided exclusion diet improves symptoms and rectal compliance in irritable bowel syndrome. Scand J Gastroenterol 2005;40:800-807.

14. Stapel SO, Asero R, Ballmer-Weber BK, et al. Testing for IgG4 against foods is not recommended as a diagnostic tool: EAACI Task Force Re- 
port. Allergy 2008;63:793-796.

15. Song KH, Jung HK, Min BH, et al. Development and validation of the Korean Rome III questionnaire for diagnosis of functional gastrointestinal disorders. J Neurogastroenterol Motil 2013;19:509-515.

16. Ligaarden SC, Lydersen S, Farup PG. IgG and IgG4 antibodies in subjects with irritable bowel syndrome: a case control study in the general population. BMC Gastroenterol 2012;12:166.

17. Zuo XL, Li YQ, Li WJ, et al. Alterations of food antigen-specific serum immunoglobulins $\mathrm{G}$ and $\mathrm{E}$ antibodies in patients with irritable bowel syndrome and functional dyspepsia. Clin Exp Allergy 2007;37:823-830.

18. Böhn L, Störsrud S, Törnblom H, Bengtsson U, Simrén M. Selfreported food-related gastrointestinal symptoms in IBS are common and associated with more severe symptoms and reduced quality of life. Am J Gastroenterol 2013;108:634-641.

19. Böhn L, Störsrud S, Simrén M. Nutrient intake in patients with irritable bowel syndrome compared with the general population. Neurogastroenterol Motil 2013;25:23-30, e21.

20. Sinagra E, Pompei G, Tomasello G, et al. Inflammation in irritable bowel syndrome: myth or new treatment target? World J Gastroenterol 2016;22:2242-2255

21. Camilleri M, Gorman H. Intestinal permeability and irritable bowel syndrome. Neurogastroenterol Motil 2007;19:545-552.

22. Zhou Q, Zhang B, Verne GN. Intestinal membrane permeability and hypersensitivity in the irritable bowel syndrome. Pain 2009;146:41-46.

23. Genuis SJ. Sensitivity-related illness: the escalating pandemic of allergy, food intolerance and chemical sensitivity. Sci Total Environ 2010;408:6047-6061.

24. Cai C, Shen J, Zhao D, et al. Serological investigation of food specific immunoglobulin $\mathrm{G}$ antibodies in patients with inflammatory bowel diseases. PLoS One 2014;9:e112154
25. Bentz S, Hausmann M, Piberger $\mathrm{H}$, et al. Clinical relevance of IgG antibodies against food antigens in Crohn's disease: a double-blind cross-over diet intervention study. Digestion 2010;81:252-264.

26. Atkinson W, Sheldon TA, Shaath N, Whorwell PJ. Food elimination based on IgG antibodies in irritable bowel syndrome: a randomised controlled trial. Gut 2004;53:1459-1464.

27. Guo H, Jiang T, Wang J, Chang Y, Guo H, Zhang W. The value of eliminating foods according to food-specific immunoglobulin $G$ antibodies in irritable bowel syndrome with diarrhoea. J Int Med Res 2012;40:204-210.

28. Mansueto P, D'Alcamo A, Seidita A, Carroccio A. Food allergy in irritable bowel syndrome: the case of non-celiac wheat sensitivity. World J Gastroenterol 2015;21:7089-7109.

29. Leonard MM, Vasagar B. US perspective on gluten-related diseases. Clin Exp Gastroenterol 2014;7:25-37.

30. Makharia A, Catassi C, Makharia GK. The overlap between irritable bowel syndrome and non-celiac gluten sensitivity: a clinical dilemma. Nutrients 2015;7:10417-10426.

31. Catassi C, Bai JC, Bonaz B, et al. Non-celiac gluten sensitivity: the new frontier of gluten related disorders. Nutrients 2013;5:3839-3853.

32. Sapone A, Bai JC, Ciacci C, et al. Spectrum of gluten-related disorders: consensus on new nomenclature and classification. BMC Med 2012;10:13.

33. Cooper BT, Holmes GK, Ferguson R, Thompson RA, Allan RN, Cooke WT. Gluten-sensitive diarrhea without evidence of celiac disease. Gastroenterology 1980;79:801-806.

34. Vazquez-Roque MI, Camilleri M, Smyrk T, et al. A controlled trial of gluten-free diet in patients with irritable bowel syndrome-diarrhea: effects on bowel frequency and intestinal function. Gastroenterology 2013;144:903-911, e903. 